\title{
Conhecimento da equipe de Enfermagem sobre a importância da Higiene Oral na Prevenção da PAVM*
}

\author{
Marysa Valéria Chaves Coimbra Miranda ${ }^{1}$, Fabiana Monteiro Braga Souza ${ }^{1}$
}

Resumo: O interesse pela saúde bucal vem aumentando constantemente, uma vez que muitas desordens sistêmicas advêm das falhas de higiene oral, sendo por isso imprescindível discutir tal temática. Este estudo teve como objetivo avaliar o conhecimento de equipes de enfermagem sobre a importância da higiene oral na prevenção da Pneumonia Associada a Ventilação Mecânica (PAVM). Foi realizado um estudo quantitativo através da aplicação de um questionário para 40 profissionais da área de saúde do município de Vitória da Conquista - BA. Avaliouse que a maioria dos profissionais das equipes de enfermagem (70\%) desconhecem o protocolo de higiene oral e $(42,1 \%)$ afirmaram não ser necessário um Cirurgião-dentista nas Unidades de Terapia Intensiva. Sendo assim, é imprescindível melhorar o treinamento em prol da prevenção do aparecimento da PAVM.

Palavras-chave: Enfermagem. Higiene oral. Saúde oral. Pneumonia Associada à Ventilação Mecânica.

\section{Knowledge of Nursing staff about the importance of Oral Hygiene in PAVM prevention}

\begin{abstract}
Interest in oral health is increasing constantly, because many systemic disorders arising from failures of oral hygiene is therefore essential to discuss such theme. This study aimed to evaluate the knowledge of nursing teams about the importance of oral hygiene in preventing Pneumonia associated with mechanical ventilation (PAVM). A quantitative study was carried out through the application of a questionnaire to 40 health workers of the municipality of Vitória da Conquista-BA. Most professionals evaluated nursing teams (70\%) are unaware of the oral hygiene Protocol and (42.1\%) said that they don't need a dentist in intensive care units. Therefore, it is essential to improve the training for the prevention of the emergence of the PAVM.
\end{abstract}

Keywords: Nursing. Oral hygiene. Oral health. Pneumonia associated with mechanical ventilation.

\section{Introdução}

A pneumonia associada à ventilação mecânica (PAVM) é resultado do desequilíbrio entre os mecanismos de defesa do indivíduo e o agente microbiano. A PAVM geralmente se desenvolve após 48 horas de intubação orotraqueal (GIROU, 2003). As vias aéreas são frequente objeto de contaminação por microrganismos que se instalam na via nasal, oral e

\footnotetext{
* Pneumonia Associada á ventilação Mecânica (PAVM).

${ }^{1}$ Colegiado de Odontologia, Faculdade Independente do Nordeste - Vitória da Conquista (BA), Brasil

Autora Correspondente: Marysa Valéria Chaves Coimbra Miranda. Av. Jesiel Noberto, 1065

Ap. 203. Cep:45028-492 - Candeias, Vitória da Conquista - BA. Contato: m.valeria123@ hotmail.com
} 
faríngea. No caso de pacientes internados em Unidade de Terapia Intensiva (UTI) existe uma alta incidência de infecções orais, o que gera uma preocupação ainda maior com relação a periculosidade da contaminação. Uma das questões mais citadas com relação a presença de bactérias gram-negativas nesta área se dá através da higiene oral (HO) deficiente (MEINBERG et al., 2012; VILELA et al., 2015).

Muitos estudos a respeito deste tema como os de Lorente et al. (2012) e Özçaka et al. (2012) trazem recomendações sobre uma HO de qualidade como forma de prevenir a PAVM. O Ministério da Saúde e organizações de cunho mundial preconizam a HO como estratégia fundamental na prevenção da PAVM, principalmente em se tratando de pacientes submetidos à ventilação mecânica.

Neste contexto, Wakiuchi et al. (2014) e Prylynski et al. (2013) destacam em seus estudos que os cuidados de enfermagem na unidade de terapia intensiva (UTI) são de grande relevância, uma vez que os pacientes admitidos sob cuidados intensivos necessitam de uma atenção maior no que diz respeito aos cuidados de saúde oral, pois na falta deles, infecções podem se instalar e gerar pneumonia. Além disso, esses podem apresentar comprometimento da função motora e/ou cognitiva, sendo crucial um atendimento adequado oferecido pelos profissionais de saúde.

Dentre as atribuições da equipe de enfermagem na UTI está a manutenção da HO. Nos pacientes com ventilação mecânica esses cuidados são imprescindíveis, pois há uma maior desidratação da cavidade oral associada a um forte odor, afinal a boca fica aberta a maior parte do tempo devido a presença do tubo endotraqueal, facilitando a colonização de bactérias e patógenos (JONES et al., 2010).

Neste contexto é válido ressaltar que na UTI, o cuidado de higiene oral geralmente é distribuído como responsabilidade da equipe de enfermagem, supervisionado pelo Cirurgião dentista (quando presente nestas unidades) ou por enfermeiros e médicos responsáveis. Contudo, não costuma se priorizar tal procedimento na rotina desta equipe, seja pela falta de comprometimento dos indivíduos envoltos no processo, pela falta de implementação de um protocolo de $\mathrm{HO}$ ou outros fatores (CUCCIO et al., 2012).

Interessante destacar que a higiene bucal é avaliada na literatura como forma de prevenção da PAVM, sendo essas práticas preventivas essenciais para a melhoria da saúde dos pacientes internados. Diante disso, há uma preocupação com o profissional que lida com tal responsabilidade. Sendo assim, os enfermeiros devem seguir protocolos de $\mathrm{HO}$, fazer mais 
treinamentos e aderir essa prática como rotina na UTI. A preocupação com infecções bucais é fundamental, uma vez que a cavidade oral é foco primário de infecções sistêmicas em pacientes internados (FRANCO et al., 2014).

Este estudo tem como objetivo avaliar o conhecimento da equipe de enfermagem sobre higiene oral de pacientes críticos sob ventilação mecânica nas Unidades de Terapia Intensiva de um município do interior da Bahia.

\section{Método}

O presente estudo foi realizado de modo qualitativo e quantitativo no que diz respeito à natureza, e quanto aos objetivos de forma exploratória. Quanto aos procedimentos técnicos é bibliográfico. O estudo exploratório realiza o estudo da viabilidade da aplicação dos métodos, da discussão dos problemas e das possibilidades de ação é realizado através da coleta dos dados para se realizar o diagnóstico e escolha do pressuposto teórico que dará suporte à investigação e elaboração do diagnóstico.

Segundo Minayo (2008) uma pesquisa qualitativa, tem caráter exploratório, sendo utilizada para obter percepções e entendimento sobre a natureza geral de uma questão, abrindo espaço para a interpretação. A quantitativa engloba o método científico que utiliza diferentes técnicas estatísticas para quantificar opiniões e informações para um estudo.

A coleta de dados foi feita através de um questionário específico estruturado pelos pesquisadores (Âpendice A), contendo 20 questões objetivas. Os questionários foram aplicados em uma amostra de 40 profissionais de saúde que englobam as equipes de enfermagem das Unidades de Terapia Intensiva de três hospitais da cidade de Vitória da Conquista -Ba.

Após a aprovação do Comitê de Ética em Pesquisa com Seres Humanos da Faculdade Independente do Nordeste com o número do CAAE 6573517.5.0000.5578 e parecer 2.077.302 obedecendo a resolução brasileira do Conselho Nacional de Saúde CNS n 466/12 que dispõe sobre as Diretrizes e Normas Regulamentadoras de Pesquisas envolvendo Seres Humanos, os participantes consentiram em fazer parte da pesquisa e iniciou-se a coleta de dados em três UTIs de Vitória da Conquista - BA.

A análise dos dados foi realizada através de artigos científicos que serviram como base para uma revisão bibliográfica. Os artigos estavam presentes nas bases de dados Scielo, 
Pubmed, Medline e Lilacs, publicados entre os anos de 2010 e 2017. Para a confecção da revisão, foram localizadas no total 30 referências (artigos, teses e dissertações) das quais foram selecionadas 23 que serviram de base para o presente trabalho.

\section{Resultados}

A amostra foi composta por 40 profissionais, com idades variando de 21 a 50 anos. A maioria desses profissionais era do sexo feminino (70\%), com até 5 anos de profissão $(22,5 \%)$ e trabalhava na UTI por um período de 5 anos ou menos. Com relação ao nível de escolaridade a maioria dos profissionais possuía $2^{\circ}$ grau completo $(72,5 \%)$. (Tabela 1$)$

Tabela 1. Perfil sociodemográfico da equipe de enfermagem. Vitória da Conquista, Bahia, Brasil, 2018.

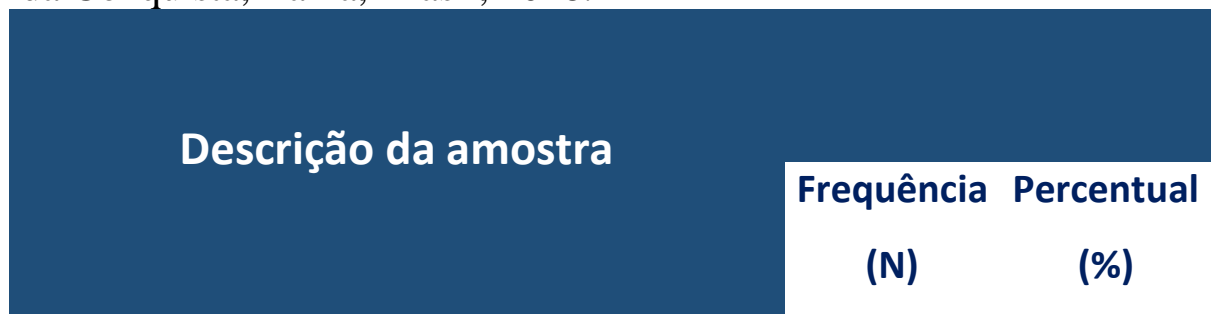

Sexo

Masculino

Feminino

28

70

Idade

21 a 26 anos

15

27 a 32 anos

6

35

33 a 38 anos

14

25

39 a 44 anos

10

15

45 a 50 anos

$6 \quad 15$

\section{Tempo de profissão}

De 0 a 5 anos

De 6 a 11 anos 


\section{Tempo que labora em UTI}

De 0 a 5 anos

20

50

De 6 a 11 anos

15

37,5

De 12 a 17 anos

$5 \quad 12,5$

De 18 a 24 anos

0

0

Nível de escolaridade

2ㅇ grau completo

$29 \quad 72,5$

Nível superior completo

$11 \quad 27,5$

Total

40

100

Fonte: Dados da Pesquisa.

Com relação ao conhecimento da importância da higiene oral 100\% dos profissionais entrevistados concordaram sobre a relevância desta medida preventiva. Entretanto, $70 \%$ dos avaliados desconhecem o protocolo (Tabela 2)

Tabela 2. Questões relacionadas a higiene oral. Vitória da Conquista, Bahia, Brasil, 2018.

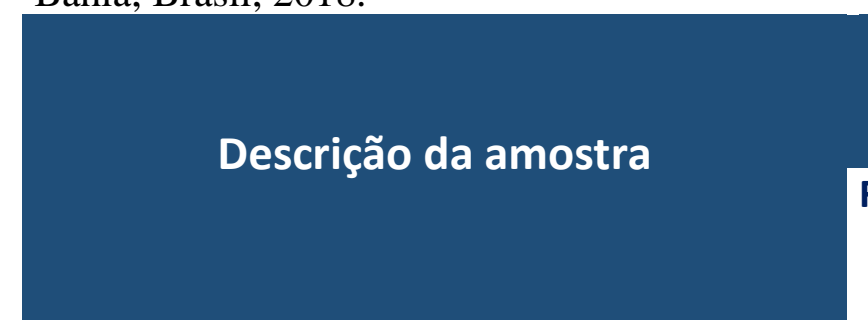

Frequência Percentual

(N)

(\%)

Importância higiene oral em pacientes críticos

Sim

40

100

Não

Não sei

0

0

0

0

\section{Conhecimento sobre o Protocolo}

Sim

$12 \quad 30$

Não

14

35

Não sei

14

35

O protocolo é adequado?

\begin{tabular}{lcc} 
Sim & 11 & 27,5 \\
Não & 0 & 0 \\
Não sei & 29 & 72,5 \\
\hline
\end{tabular}

Fonte: Dados da Pesquisa. 
Sobre como é feita a higiene oral, $22,5 \%$ dos profissionais afirmaram que é limitada aos dentes, $50 \%$ afirma que abrange língua e mucosa oral. 72,5\% disseram que existe rotina para higiene oral e que é feita de 1 a 2 vezes por dia (75\%) (Tabela 3 ).

Tabela 3. Como é feita a higiene oral. Vitória da Conquista, Bahia, Brasil, 2018.

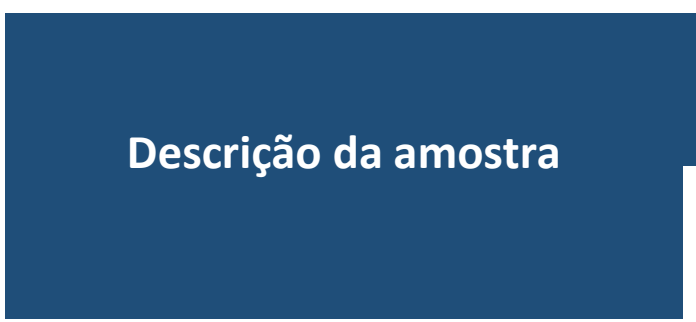

\section{Frequência}

(N)
Percentual

(\%)

\section{Limita-se aos dentes}

$\begin{array}{lcc}\text { Sim } & 9 & 22,5 \\ \text { Não } & 20 & 50 \\ \text { Não sei } & 11 & 27,5 \\ & & \\ \text { Abrange língua e mucosa oral } & & 50 \\ \text { Sim } & 20 & 22,5 \\ \text { Não } & 9 & 27,5 \\ \text { Não sei } & 11 & \end{array}$

\section{Existe rotina definida para Higiene \\ oral}

Sim

29

72,5

Não

5

12,5

Não sei

6

15

\section{Frequência}

1 vez por dia

2 vezes por dia

15

37,5

Não sei

10

25

Fonte: Dados da Pesquisa.

No que se refere a associação de bactérias com a falta de higiene oral, 72,5\% afirmaram ter relação a associação da higiene oral ao acumulo de bactérias na boca, 52,5\% relacionaram a higiene oral com o desenvolvimento da PAVM e 45\% não souberam responder em relação a higiene com outras doenças como Endocardite e Periodontite. (Tabela 4). 
Tabela 4. Associação de patologias com a higiene oral. Vitória da Conquista, Bahia, Brasil, 2018.

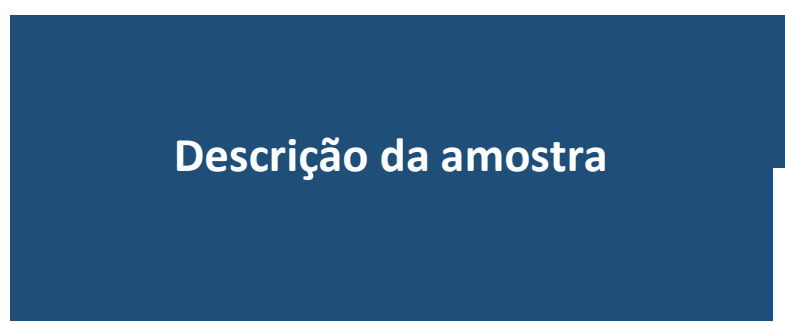

\section{Frequência Percentual}

(N)

(\%)

Associa higiene oral ao acumulo de bactérias

na boca

Sim

29

72,5

Não

11

27,5

Não sei

$0 \quad 0$

Relaciona a higiene oral com a PAVM

Sim

$21 \quad 52,5$

Não

19

47,5

Não sei

0

0

Relaciona higiene oral com Endocardite,

Periodontite e outras infeç̧ões

Sim

15

37,5

Não

7

17,5

Não sei

18

45,0

Fonte: Dados da Pesquisa.

Dos profissionais que responderam o questionário, $80 \%$ afirmam ser necessária a presença de um cirurgião dentista na UTI (Tabela 5). 
Tabela 5: Distribuição sobre necessidade de cirurgião dentista na equipe da UTI Vitória da Conquista, Bahia, Brasil, 2018.

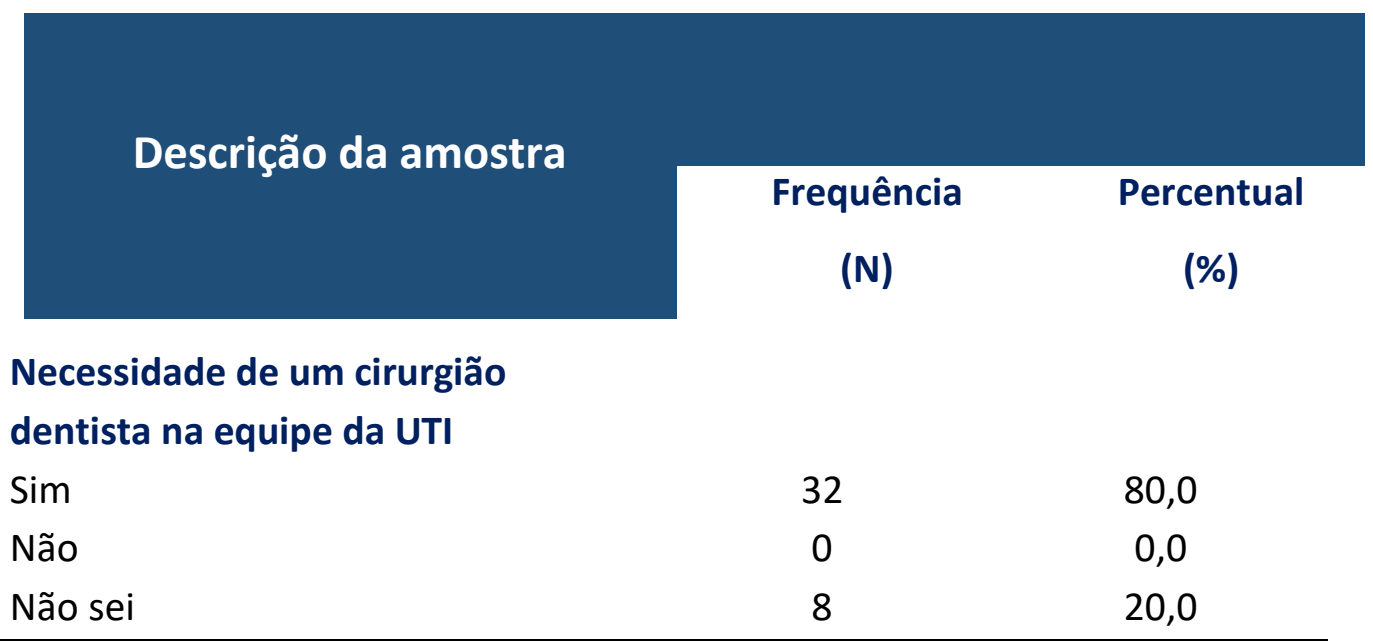

Fonte: Dados da Pesquisa.

\section{Discussão}

Pinheiro e Almeida (2014) ao tratar sobre Odontologia Hospitalar em Unidades de Terapia Intensiva (UTI) destacam que a incidência de microrganismos neste ambiente apresenta um potencial patogênico ainda maior e que acarreta uma frequência alta de infecções, por isso, atitudes preventivas devem ser postas em prática em centros hospitalares, já que há agravamentos frequentes desta problemática e isso emerge uma preocupação latente que incide em diversos protocolos específicos para intervenção deste problema.

Seguir protocolos sobre a higiene oral (HO) é fundamental para evitar contaminações, pois as normas e condutas de higiene oral quando aplicadas com frequência tornam-se preventivas, são mais eficientes e corroboram para redução das comorbidades associadas às alterações orais em UTI (FRANCO et al., 2014).

Jones et al. (2011) destaca que o protocolo deve ser realizado pelo Cirurgião-dentista diretamente para a equipe de enfermagem, iniciando com medidas que vão desde o posicionamento do paciente até as medidas de procedimento de higiene oral.

Este estudo averiguou também que a higiene oral não deve limitar-se aos dentes, deve abranger toda a mucosa oral, devendo ter uma frequência adequada. Silveira et al. (2010) salienta que o procedimento não ocorre como deveria, nem na frequência adequada, o que foi 
corroborado neste presente estudo, sendo interessante que se incentive o aumento da frequência do cuidado deste tipo de higiene (NOGUEIRA; JESUS, 2017).

Orlandini e Lazzari (2012) destacaram em estudo feito que um terço da equipe de enfermagem analisada considera que a frequência da higiene oral deve ser melhorada, englobando a escovação dos dentes, além do uso da solução contendo clorexidina.

Neste estudo viu-se que todos alegaram que a Clorexidina é usada, 70\% destacaram que é feito o uso de gaze estéril, porém a grande maioria alega que a escova usada não é descartável ou não sabem se é.

Wong et al. (2016) por sua vez destaca que a técnica de higienização bucal usada por equipes de enfermagem é de gaze embebida em solução antisséptica, geralmente 2 vezes ao dia. Neste estudo viu-se que 67,5\% dos questionados destacam que a escova de dente é utilizada, mas esta não é descartada na maior parte dos casos.

Estudo feito por Cuccio et al. (2012) avaliou a escovação dentária associada ao uso da clorexidina salientando que essa técnica de higiene oral feita quatro vezes ao dia gera uma redução significativa nos índices de PAVM.

Caldeira (2011) cita que a solução de clorexidina é um antimicrobiano barato e de fácil aplicação, além de ter um baixo nível de reações adversas, sendo eficiente para combater bactérias comuns em ambientes hospitalares.

A PAVM, Endocardite, Periodontite e outras infecções, poderiam ser evitadas através da higiene oral, o que é confirmado por muitos outros estudos como de Orlandini e Lazzari (2012), Meinerg et al. (2012), Vilela et al. (2015) e Lorente et al. (2012).

As equipes de enfermagem das UTIS analisadas não são treinadas para este trabalho, e a maioria afirmou não procurar capacitação e treinamento. Nota-se que a frequência da realização de higiene também não é adequada e conforme estudo de Özçaka et al., (2012) enfrenta muitas divergências, que pode se dar pela existência de complicações graves nas UTIs como risco de mort e problemas neurológicos, que não permitem um protocolo do número de vezes que a higiene oral é feita.

Ressalta-se a importância de efetivar protocolos baseados nas atuais evidências que primem por uma atuação interdisciplinar entre enfermagem e odontologia para atender as necessidades do paciente, sendo extremamente relevantes para a prática clínica (WAKIUCHI et al., 2014). 
Além disso, é necessário um melhor desempenho do profissional em prol da HO na UTI, pois a HO é uma prática extremamente importante na assistência a pacientes, e para aqueles que em UTI fazem uso de ventilação mecânica a preocupação é ainda maior. A HO deve ser implementada como rotina, sendo necessária uma equipe competente e eficiente na função de prevenir complicações (PRYLYNSKI et al., 2013).

É importante que a equipe de enfermagem tenha uma preocupação crescente com o aperfeiçoamento do protocolo de higiene oral, tornando estas ações mais eficientes e corroborando para redução das comorbidades associadas às alterações orais UTIs (GOMES; SILVA, 2010).

Neste contexto vale destacar que o questionário avaliou corretamente sobre a higiene bucal e seus efeitos na prevenção da PAVM. Nota-se que o mais interessante é que sejam utilizadas ações preventivas afim de evitar a infecções respiratórias (PERES, 2014; PINHEIRO; ALMEIDA, 2011).

Caso não seja feita uma higiene oral adequada aumentaria a concentração de patógenos na saliva, que por sua vez podem ser aspirados pelo pulmão de modo a afetar a sua imunidade (NOGUEIRA; JESUS, 2017). A prevenção da pneumonia hospitalar em UTI pode diminuir as taxas de mortalidade (SOUZA et al., 2013).

Vilela et al. (2015) alerta para os problemas que a falta de um Cirurgião-dentista na UTI pode causar, pois é o mais capacitado para alertar sobre a higienização oral, evitando que o paciente tenha uma maior colonização do biofilme dental, contribuindo para a prevenção de infecções hospitalares. Por sua vez, Robertson e Cartes (2013) abordam em seu estudo que este profissional trabalhando em UTI é mais fácil identificar e tratar várias doenças que acometem a região oral e atuar na prevenção para evitar complicações para o paciente.

\section{Conclusão}

As manobras de higiene oral bem conduzidas tornam-se preventivas e eficientes na redução das comorbidades associadas às alterações orais em Unidade de Terapia Intensiva (UTI). Nota-se, a partir do estudo que é essencial investir em maior conhecimento e treinamento da equipe de enfermagem que atua diretamente na higiene bucal de pacientes entubados em UTI, para prevenir de forma eficaz a Pneumonia Associada à Ventilação Mecânica. Percebe-se 
ainda que o cirurgião-dentista, profissional com competência na área de higiene bucal, ao fazer parte da equipe de saúde da UTI poderia reduzir a ocorrência de infecção oral e, consequentemente sistêmica, o que conduziria a uma menor permanência do paciente no tratamento intensivo.

\section{Referências}

CALDEIRA, P. M. Higiene oral de pacientes em intubação orotraqueal internados em uma unidade de terapia intensiva. Revista Enfermagem Integrada. Ipatinga, v.4, n. 1, p. 731-41, 2011.

CUCCIO, L; CERULLO, E.; PARADIS, H.; PADULA, C.; RIVET, C. STEEVES, S, LYNCH J. An evidence-based oral care protocolto decrease ventilator-associated pneumonia. Dimens Crit Care Nurs, v. 31, n. 5, p. 301-8, 2012.

FRANCO et al. Higiene bucal para pacientes entubados sob ventilação mecânica assistida na unidade de terapia intensiva: proposta de protocolo. Arq Med Hosp Fac Cienc Med. São Paulo, v. 59, n. 3, p.126-31, 2014.

GODOY, A. C. F.; VIEIRA, R. J.; CAPITANI, E. M. Alteração da pressão intra-cuff do tubo endotraqueal após mudança da posição em pacientes sob ventilação mecânica. Rev. bras. ter. intensiva, v.24, n.4, 2012.

GOMES, A. M.; SILVA, R. C. L. Prevenção da Pneumonia Associada à Ventilação Mecânica: o que Sabem os Enfermeiros a esse Respeito? J Nurs UFPE online, v. 4, n. 2, p. 605-14, 2010.

JONES, D.; MUNRO, C.; GRAP, M.; KITTEN, T.; EDMOND, M. Oral care and bacteremia risk in mechanically ventilated adults. Heart lung, v. 39, n. 6, p. 57-65, 2010.

JONES, D. J.; MUNRO, C. L.; GRAP M. J. Natural history of dental plaque accumulation in mechanically ventilated adults: a descriptive correlational study. Intensive critare nurs, v.27, n.6, p; 299-304, 2011.

PERES, M. P. S. Higiene bucal para pacientes entubados sob ventilação mecânica assistida na unidade de terapia intensiva: proposta de protocolo. Arq Med Hosp Fac Cienc Med. São Paulo, v. 59, n. 3, p. 126-31, 2014.

PRYLYNSKI, D.; PELZER, M.; SANTOS, S.; SILVA, M.; COSTA, C.; GASPARIM, A. B. Ações educativas de enfermagem em saúde bucal de idosos em uma instituição de longa permanência. Cogitar e enferm, v.14, n. 4, p. 696-702, 2013. 
MEINBERG, M. C. A.; CHEADE, M. F. M; MIRANDA, A.; FACHINI, M.; LOBO, S. M. Uso de clorexidina $2 \%$ gel e escovação mecânica na higiene bucal de pacientes sob ventilação mecânica: efeitos na pneumonia associada a ventilador. Rev bras ter intensiva, v. 24, n. 4, p. 369-74, 2012.

MINAYO, M. C. S. O Desafio do Conhecimento: Pesquisa Qualitativa em Saúde. 6 Ed. São Paulo: Hucitc, 2006.

ORLANDINI, G.M.; LAZZARI, C.M. Conhecimento da equipe de enfermagem sobre higiene oral em pacientes criticamente enfermos. Rev. Gaúcha Enfermagem, v. 33, n. 3, p. 34-41, 2012.

ÖZÇAKA, Ö.; BAŞOĞLU, O.; BUDUNELI, N.; TAŞBAKAN, M.; BACAKOĞLU, F.; KINANE, D.F. Chlorhexidine decreases the risk of ventilator-associated pneumonia in Intensive care unit patients: a randomized clinical trial. Periodontal Res, v. 47, n. 5, p. 584-92, 2012.

PINHEIRO, T.S; ALMEIDA, T.F. A saúde bucal em pacientes de uti. Rev Baiana de Odontologia, v. 5, n. 2, p. 94-103, 2014.

LORENTE, L.; LECUONA, M.; JIMÉNEZ, A.; PALMERO, S.; PASTOR, E.; LAFUENTE, N. Ventilator-associated pneumonia with or without tooth brushing: a randomized controlled trial. Eur J Clin Microbio Infect Dis, v. 31, n. 10, p. 2621-9, 2012.

NOGUEIRA, J. W. S; JESUS, C. A. C. Higiene bucal no paciente internado em unidade de terapia intensiva: revisão integrativa. Rev. Eletr. Enf, v 1, n.1, 2017.

ROBERTSON, T.; CARTER, D. Oral intensity: reducing non-ventilator-associated hospitalacquired pneumonia in care dependent, neurologically impaired patients. Can J Neurosci Nurs, v. 35, n. 2, p. 10-7, 2013.

SILVEIRA, I. R.; MAIA, F.; GNATTA, J. R.; LACERDA, R. A. Higiene bucal: prática relevante na prevenção de pneumonia hospitalar em pacientes em estado crítico. Acta Paul Enferm, v. 23, n. 5, p.697-700, 2010.

SOUZA, A. F.; GUIMARÃES, A.C.; FERREIRA, E. F. Avaliação da implementação de novo protocolo de higiene bucal em um centro de terapia intensiva para prevenção de pneumonia associada à ventilação mecânica. Rev Min Enferm, v. 17, n. 1, p. 177-84, 2013.

VILELA, M.; FERREIRA, G.; SANTOS, P. REZENDE, N. M. Oral care and nosocomial pneumonia: a systematic review, v. 13, n. 2, p. 290-6, 2015.

WAKIUCHI, J.; FONTES, M.; PAPA, M.A. Oral hygiene in patients under mechanical ventilation: an integrative review. Journal of Nursing UFPE, v. 8, n. 7, p. 97-103, 2014.

WONG, T.; SCHLICHTING, A. B.; STOLTZE, A. J.; FUllER, B. M.; PEACOCK, A. HARLAND, K. K. No Decrease in Early Ventilator Associated Pneumonia After Early Use of Chlorhexidine. Am J Crit Care, v. 25, n. 2, p. 173-7, 2016. 


\section{Como citar este artigo (Formato ABNT):}

MIRANDA, Marysa Valéria C.C.; SOUZA, Fabiana Monteiro B. Conhecimento da equipe de Enfermagem sobre a importância da Higiene Oral na Prevenção da PAVM. Id on Line Rev.Mult. Psic., 2018, vol.12, n.40, p.1209-1221. ISSN: 1981-1179.

Recebido: 28/05/2018

Aceito 30/05/2018 